\title{
Fabrication of high-density nanostructures by electron beam lithography*
}

\author{
O. Dial, C. C. Cheng, ${ }^{\text {a) }}$ and A. Scherer \\ Electrical Engineering, California Institute of Technology, Pasadena, California 91125
}

(Received 29 May 1998; accepted 29 September 1998)

\begin{abstract}
We demonstrate a fabrication method to define high-density, uniform nanostructures by electron beam lithography at conventional beam voltages $(<40 \mathrm{kV})$. Here we optimize the exposure and development conditions needed to generate such nanostructure arrays using polymethylmethacrylate as positive resist and isopropyl alcohol as a developer. Arrays of $12 \mathrm{~nm}$ dots with $25 \mathrm{~nm}$ period and $20 \mathrm{~nm}$ lines with $40 \mathrm{~nm}$ period were fabricated to show the resolution of this optimized process.

(C) 1998 American Vacuum Society. [S0734-211X(98)19106-0]
\end{abstract}

\section{INTRODUCTION}

Advances in new fabrication technologies have enabled us to miniaturize the lateral dimensions of devices to far below $100 \mathrm{~nm} .{ }^{1}$ Dense and uniform arrays of such features are becoming increasingly important in high-density magnetic storage media, ${ }^{2,3}$ dense subwavelength optical gratings, metal-semiconductor-metal photodetectors, electronic surface superlattice devices ${ }^{4,5}$ as well as for templates for epitaxial regrowth on patterned substrates. Lithography is usually the most critical procedure for successfully defining structures with such dimensions. ${ }^{6} 50 \mathrm{~nm}$ period structures have so far been obtained by various state of the art direct e-beam lithography approaches in which proximity effects can be minimized. For example, by using high voltage electron beam systems ${ }^{7-9}$ or inorganic electron beam resists are found to provide high contrast and resolution. However, the high required doses and restrictive sample geometries, as well as the difficulty of integrating new inorganic materials into practical nanofabrication procedures have so far limited the application of these techniques in defining useful samples. Here we describe a method using single-level polymethylmethacrylate (PMMA) resist to define structures with $\sim 10 \mathrm{~nm}$ feature size by conventional electron beam voltages $(<40 \mathrm{kV})$ obtained with a field-emission scanning electron microscope. Our method relies on using a high contrast PMMA developer: isopropyl alcohol (IPA), and carefully controlling the electron dose and spot size. Although PMMA development in IPA and IPA: $\mathrm{H}_{2} \mathrm{O}$ mixtures has been used before for higher contrast and sensitivity, ${ }^{10}$ the ultimate pattern resolution was not explored in detail. We have also optimized both exposure and development conditions to explore the limitations in pattern density which can be obtained with this process.

\section{PROCEDURE}

\section{A. Electron beam lithography system}

A high resolution electron beam lithography system requires a stable electron source and a good electron optical column to obtain a small beam size. Commercial scanning

\footnotetext{
*No proof corrections received from author prior to publication.

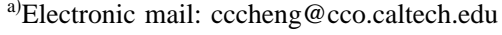

electron microscopes (SEMs) have been developed for high resolution imaging, and can meet these requirements. These instruments can readily be modified into high resolution electron beam writers. ${ }^{8}$ We have chosen a Hitachi S-4500II SEM equipped with a cold field emitter. This SEM can be used to obtain a resolution of about $1 \mathrm{~nm}$ at $20 \mathrm{kV}$ and $3 \mathrm{~nm}$ at $1 \mathrm{kV}$. The field-emission tip has several well-known advantages over thermionic emitters, such as the low chromatic aberration and high brightness. The disadvantage of this emission source lies in the variation of the beam current, which has so far limited its application for electron beam lithography. If the beam current is periodically measured during the beam-writing procedure, however, this drift can be compensated for with the beam-writing software. For this purpose, we have developed an advanced beam-writing package which allows dynamic control over the microscope functions, such as focus, magnification, sample position, beam blanking, and image acquisition. The beam blanking is done by electrostatically deflecting the beam over an aperture. Our computer system also measures the beam current through the floating objective aperture within the column as well as a Faraday cup located next to the sample. This aperture current was observed to be directly proportional to the current measured at the sample, although the constant of proportionality is a function of column pressure. During the lithography procedure, we periodically monitor the aperture current and compensate for drift in the emitter brightness by adjusting the beam dwell time. With this method, we have converted this high resolution instrument into a state of the art lithographic tool. The beam position within our lithography system is controlled by applying external voltage signals from a 16-bit digital/analog board onto the beam deflection yokes at speeds of up to 100000 pixels/s. Programs have been written to translate standard computer aided design files into vector-scan format which enables the definition of patterns with arbitrary shapes on the sample.

\section{B. Sample preparation}

We used silicon and GaAs wafer substrates which were coated with a $15 \mathrm{~nm}$ layer of $\mathrm{Au}-\mathrm{Pd}$ by magnetron direct current (dc) sputter deposition. A $40 \mathrm{~nm}$ layer of $950 \mathrm{~K}$ molecular weight PMMA dissolved in chlorobenzene was spun onto these metallized substrates. The PMMA was then 


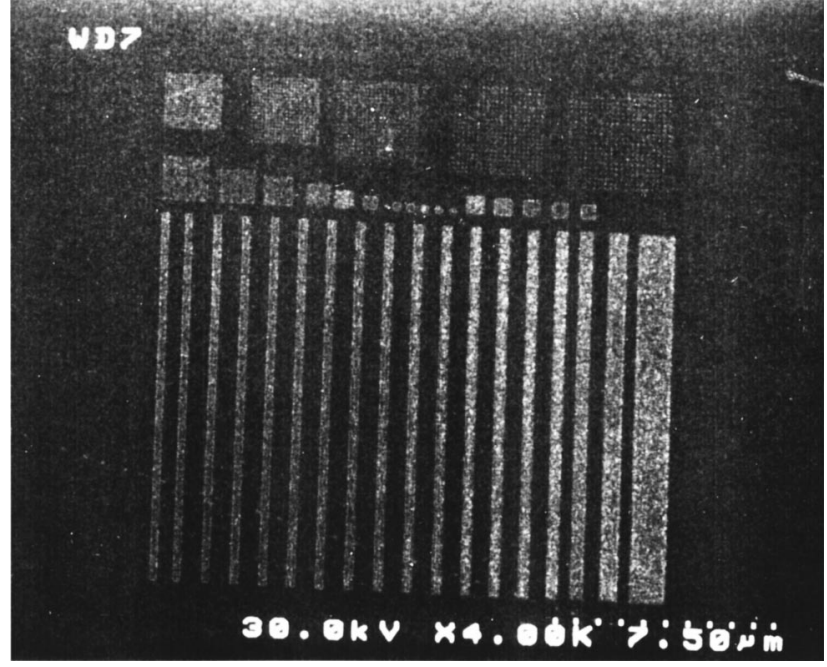

FIG. 1. Test pattern used for determining the optimal dose and minimum line and dot spacing for a given development condition. Note the five finely spaced gratings for accurate determination of resolution at near-optimum conditions.

oven baked at $150{ }^{\circ} \mathrm{C}$ for $24 \mathrm{~h}$. Immediately before the exposure, the PMMA was again hot-plate baked at $180{ }^{\circ} \mathrm{C}$ for 2 min to further improve the adhesion of the polymer layer.

A test pattern which includes several line and dot arrays with different periods and exposure doses was generated and beam written onto the sample. Figure 1 shows a SEM picture of this test pattern after development. Exposures were performed at 30 and $15 \mathrm{kV}$ using beam currents of 5 and $10 \mathrm{pA}$, respectively. Within this test pattern, the writing speed was selected such that exposure dose was varied from 4.0 to 12.0 $\mathrm{mC} / \mathrm{cm}^{2}(0.01$ to $0.03 \mathrm{pC} / \mathrm{dot})$ for both voltages when exposing a series of dot arrays. The center to center spacing of the dots was changed systematically in a series of patterns, with the intent of determining the most dense pattern which could be still resolved for any given exposure and development condition. Similar patterns were used to evaluate the optimum line exposure conditions, and to determine the best conditions for defining dense line gratings.

After the exposure, some of the samples were developed for different times in pure IPA at room temperature. The optimal developing time for this procedure was determined, and the minimum feature size was compared with samples developed in our standard developer, a 3:7 mixture of cellusolve and methanol. The accuracy within which we could control the development time was approximately $\pm 1 \mathrm{~s}$. Once the samples were developed, the electron beam-written pattern was transferred from the PMMA resist into the $\mathrm{Au}-\mathrm{Pd}$ sputter-deposited layer by using argon ion milling for $45 \mathrm{~s}$ with a $10 \mathrm{~mA}$ ion beam at $1500 \mathrm{~V}$. This allowed us to obtain high contrast images of the resulting patterns. We also performed lift-off processes on some samples to confirm the suitability of the optimal lithographic conditions for metal deposition and additive processing. After fabrication, the samples were carefully inspected in the Hitachi S-4500 SEM, and the sizes and quality of the resulting structures were determined. (a)

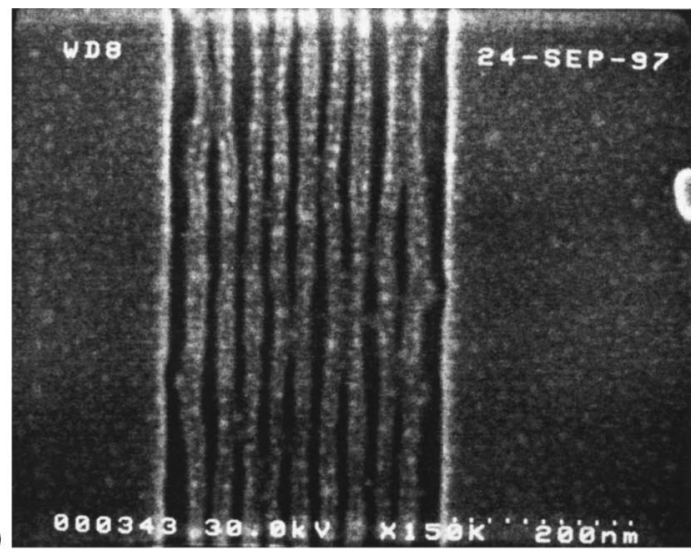

(b)

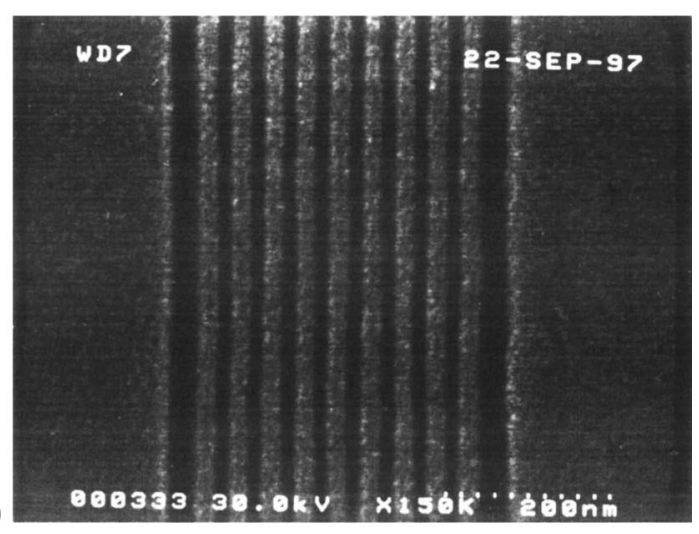

FIG. 2. (a) SEM micrograph of line grating after showing delamination of the PMMA resist during development. (b) SEM micrograph of a grating with $20 \mathrm{~nm}$ lines and $40 \mathrm{~nm}$ pitch.

\section{RESULTS AND DISCUSSION}

\section{A. Resist adhesion}

There are several important factors which determine the quality of the lithographic process. The size of the electron beam, the exposure dose, the development time, the developer contrast, and the adhesion of the PMMA have been optimized in this study. When PMMA is oven baked at $150{ }^{\circ} \mathrm{C}$ even for over $24 \mathrm{~h}$, delamination of the PMMA often takes place during the development procedure. Figure 2(a) shows a typical example of this problem. Here we can observe a $\sim 40 \mathrm{~nm}$ period line grating sample, exposed at 0.9 $\mathrm{nC} / \mathrm{cm}$, after development and milling through the AuPd, and bridges can be seen connecting the lines. This problem can be corrected through the introduction of an additional hotplate baking step immediately before beam writing. Figure 2(b) shows a SEM micrograph of a similar pattern with 20 $\mathrm{nm}$ lines and $40 \mathrm{~nm}$ period after this adhesion step was included in the procedure. As can be seen in this micrograph, the quality of the lines is improved significantly, and the delamination problem is no longer significant. In all of the following work, we therefore have added this hot-plate baking step to the fabrication procedure.

\section{B. Optimization of exposure and development}

Figure 3 shows a plot of the minimum observed dot array pitch versus development time for both 30 and $15 \mathrm{kV}$ expo- 


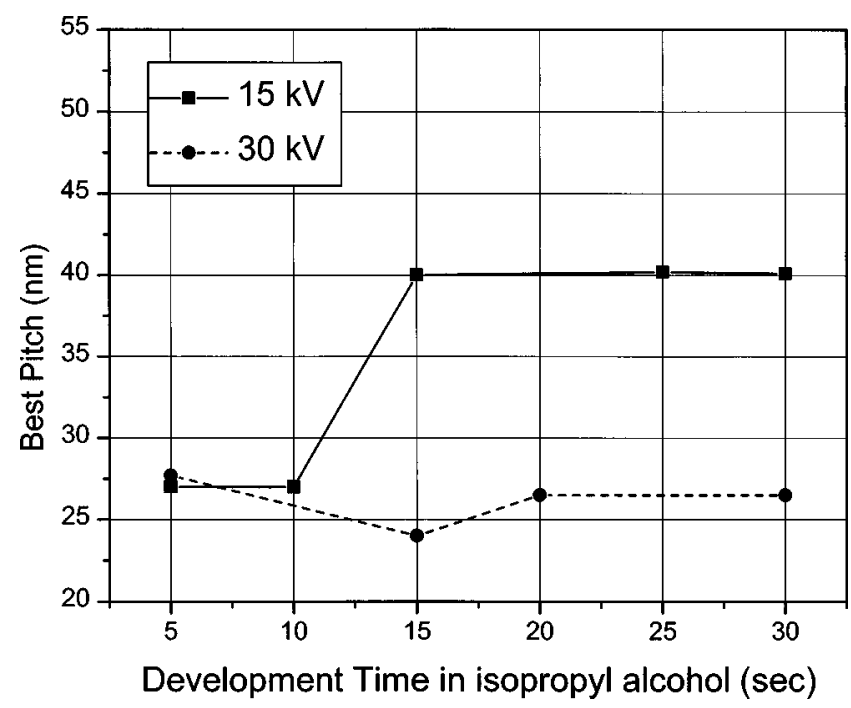

FIG. 3. Plot of the minimum pitch vs development time in IPA.

sures. Each one of the data points in this plot is representative of a separate test pattern, developed for a different time. We then determined the optimal electron dose and pitch within each developed test pattern. When beam writing at 15 $\mathrm{kV}$, we observe a monotonic decrease in the smallest resolvable pitch as the development time is decreased. There is an abrupt jump at approximately $15 \mathrm{~s}$ where the minimum observed resolution moves from a coarse set of arrays to a set for finer determination of pitch (see Fig. 1). When a $30 \mathrm{kV}$ electron beam is used for exposure, a minimum dot array pitch is obtained for an optimum development time of $15 \mathrm{~s}$. The corresponding optimal dose for this condition is 5 $\mathrm{mC} / \mathrm{cm}^{2}$, when assuming a $100 \mathrm{~nm}^{2}$ exposed area. We find that this exposure dose is significantly higher than the conditions we use for developing in cellusolve:methanol mixtures. When using this condition, dot arrays with periods of 25 and $30 \mathrm{~nm}$ are reproducibly obtained, and Figs. 4(a) and 4(b) show SEM micrographs of these. Within these arrays, we find that the diameters of the developed and ion milled holes range from 10 to $12 \mathrm{~nm}$.

Figure 5 shows a plot of the minimum pitch obtained versus the exposure dose, when optimizing the development time. In this case, we only plot data for $30 \mathrm{kV}$ exposures. The minimum observed period again monotonically decreases with the exposure dose. Below the lowest doses plotted in Fig. 5, pattern irregularities and missing dots are observed before the patterns disappear completely. It should be noted here that all of the patterns are inspected after argon ion milling, which requires the PMMA to be completely developed leaving a relatively clean bottom.

\section{Lift-off processing}

In order to confirm the complete removal of PMMA in the exposed areas, we have also conducted lift-off experiments on our patterns. For these, we deposit thin layers $(\sim 20 \mathrm{~nm})$ of $\mathrm{Au}$ and $\mathrm{Au}-\mathrm{Pd}$ by vapor deposition and dc magnetron sputter deposition, respectively. After deposition of the

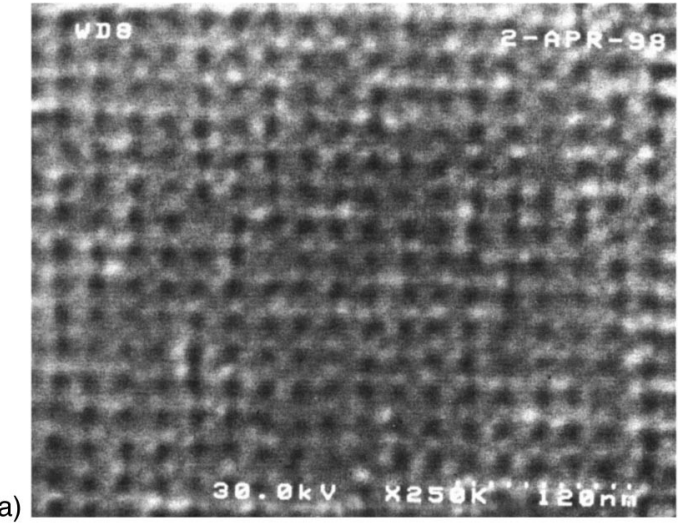

(b)

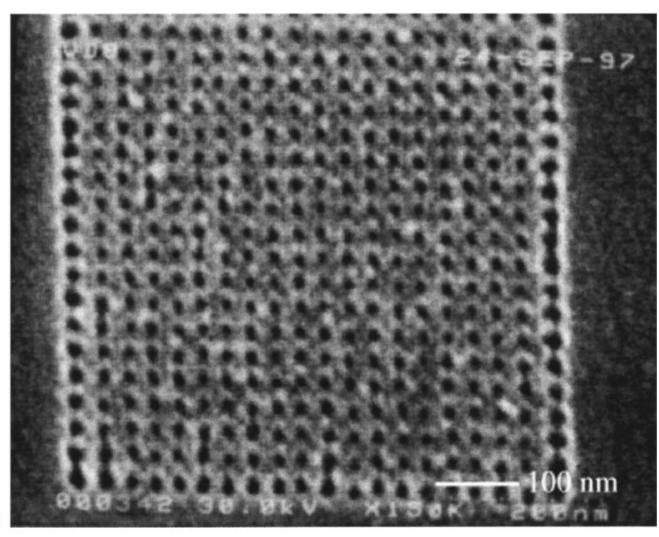

FIG. 4. (a) SEM micrograph of a $25 \mathrm{~nm}$ period dot array on etched through a AuPd layer on Si obtained at the optimal exposure and development condition. (b) SEM micrograph of a $30 \mathrm{~nm}$ period dot array.

metal, the lift-off procedure consisted of a simple acetone rinse, which completely dissolved the PMMA resist. Again, we observe high fidelity in the resulting nanofabricated patterns, as can be seen in Figs. 6(a) and 6(b). Figure 6(a) shows a lift-off sample after sputter deposition of $\mathrm{Au}-\mathrm{Pd}$ into a 40 $\mathrm{nm}$ period short line grating on GaAs. In this case, some

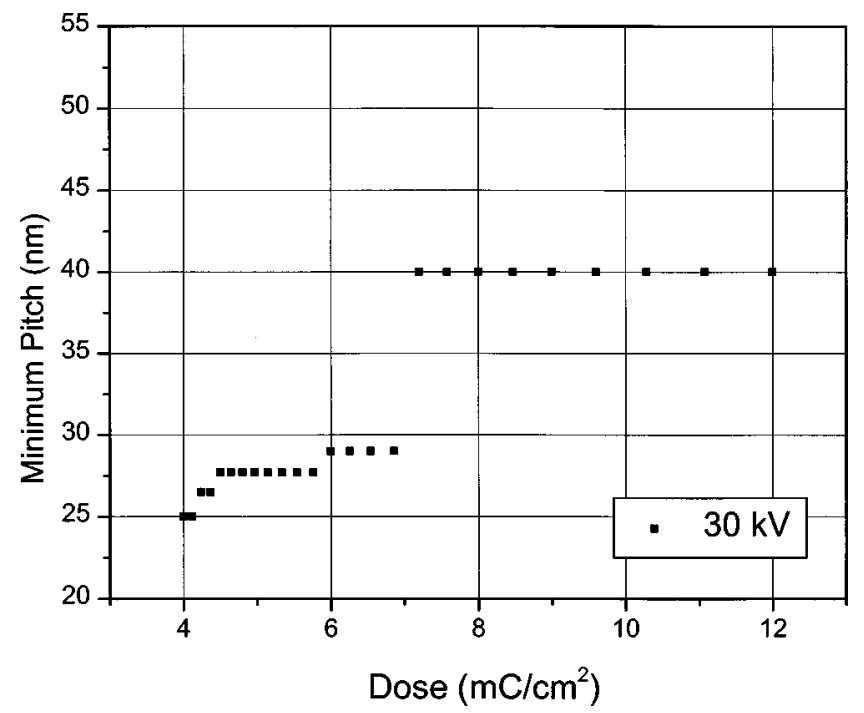

FIG. 5. Plot of the minimum pitch obtained in dot arrays vs the exposure dose at optimal development conditions. 

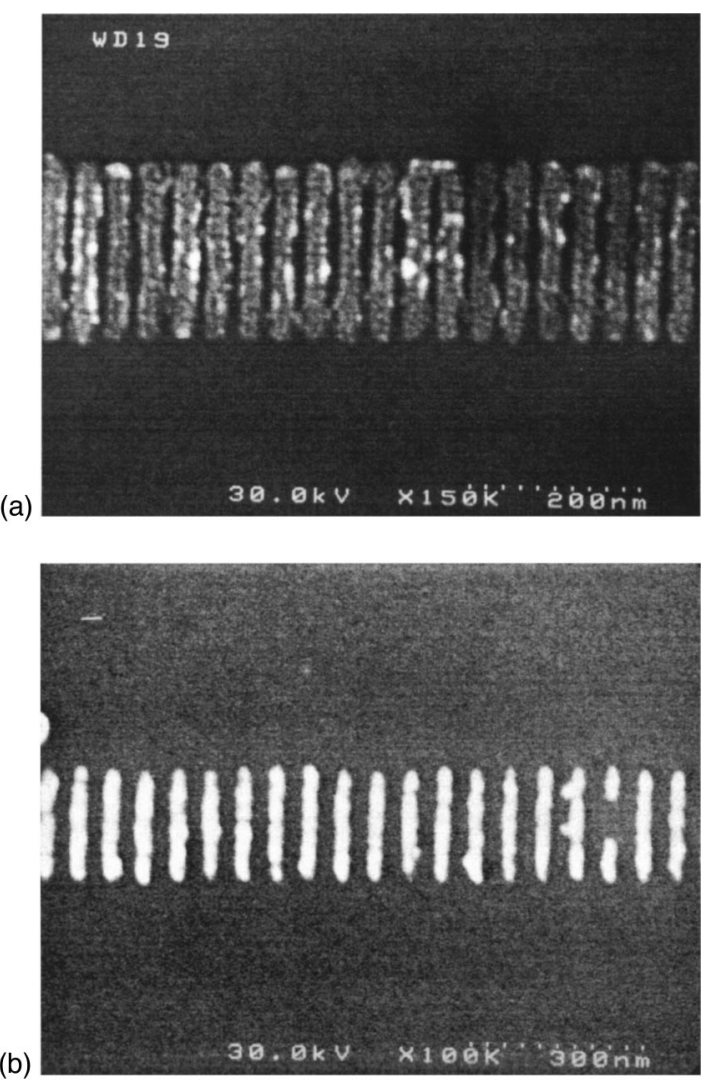

FIG. 6. (a) Lift-off pattern revealing $40 \mathrm{~nm}$ period AuPd particles deposited by dc sputter deposition. (b) Lift-off pattern showing $60 \mathrm{~nm}$ period Au particles deposited by thermal evaporation.

flags are seen, which result from the conformal deposition procedure. Figure 6(b) shows a SEM micrograph of a $60 \mathrm{~nm}$ period short line grating consisting of $20 \mathrm{~nm}$ wide and 200 $\mathrm{nm}$ long Au bars, again deposited directly onto a GaAs sub- strate. In this case, no flags were observed since we used thermal evaporation, a more directional deposition process.

\section{CONCLUSIONS}

We have demonstrated that it is possible to use PMMA resist to obtain very dense arrays of nanostructures. These were defined by electron beam lithography in a cold fieldemission scanning electron microscope, and developed in IPA. The resulting combination of very small electron beam diameter and very high contrast developer allows us to extend the resolution of PMMA resist to $\sim 10 \mathrm{~nm}$ feature sizes with high density and uniformity. By using low electron energies and a high brightness source, we are also able to increase the electron beam-resist interaction and improve the speed of the electron beam writing process. We believe that the simple optimization described here can still be further optimized, but indicates that PMMA is a reliable lift off and etch mask even at these dimensions.

\section{ACKNOWLEDGMENTS}

This work was supported by the Army Research Office and the National Science Foundation, which are gratefully acknowledged.

${ }^{1}$ B. P. Van der Gaag and A. Scherer, Appl. Phys. Lett. 56, 481 (1990).

${ }^{2}$ S. Y. Chou, P. R. Krauss, and P. I. Renstrom, Appl. Phys. Lett. 66, 3114 (1995).

${ }^{3}$ R. M. H. New, R. F. W. Pease, and R. L. White, J. Vac. Sci. Technol. B 13, 1089 (1995).

${ }^{4}$ B. Houli, V. Umansky, and M. Hciblum, Semicond. Sci. Technol. 8, 490 (1993).

${ }^{5}$ A. Scherer and B. P. Van der Gaag, Proc. SPIE 1284, 149 (1990).

${ }^{6}$ R. E. Behringer, P. M. Mankiewich, and R. E. Howard, J. Vac. Sci. Technol. B 5, 326 (1987).

${ }^{7}$ A. Scherer and H. G. Craighead, Appl. Phys. Lett. 49, 1284 (1986).

${ }^{8}$ W. Chen and H. Ahmed, J. Vac. Sci. Technol. B 11, 2519 (1993).

${ }^{9}$ W. Chen and H. Ahmed, J. Vac. Sci. Technol. B 13, 2883 (1995).

${ }^{10}$ M. A. Moshin and J. M. G. Cowic, Polymer 29, 2130 (1988). 